\title{
A PROBABLE MECHANISM OF REPEATED EXPLOSIONS OF COMPACT OBJECTS
}

\author{
L. M. OZERNOY \\ P. N. Lebedev Physical Institute, Academy of Sciences of the U.S.S.R., \\ Moscow, U.S.S.R.
}

\begin{abstract}
This paper considers a magnetoid model for nuclei of compact objects in which repeated explosions are caused by instability in twisted magnetic fields. The derived frequency and energy of individual explosions agrees with observations.
\end{abstract}

Lately more and more theorists and observers have inclined to the opinion that galaxy and quasar nuclei contain, in addition to stars, a magnetoid, i.e. a supermassive plasma body, the equilibrium and stability of which are determined by internal motions of a rotational type and magnetic fields. The elementary magnetoid theory, developed several years ago (Ozernoy, 1966; 1968), offered the possibility of explaining semiquantitatively the two fundamental properties of quasars and quasar-like phenomena in galactic nuclei, that is, the powerful energy output from a small volume and the variability of this radiation. Recently we have obtained new results concerning three relevant topics: (a) magnetoid structure and evolution (Ozernoy and Usov, 1971); (b) the mechanism of repeated explosions (Ozernoy and Somov, 1971); and (c) the role of magnetoid explosions in the formation of stars and star systems (Ozernoy, 1971). These results give a more complete theoretical picture of quasi-stellar phenomena.

My purpose is to describe briefly the mechanism of repeated explosions, which probably operates in the nuclei of galaxies and quasars leading to ejection of clouds and jets of relativistic plasma.

The idea of this mechanism is the following: Let us imagine a supermassive rotating star, connected with the surrounding plasma by a magnetic field, inclined to the axis of rotation. As a result of the rotation the magnetic field is twisted. When rot $\mathbf{H}$ increases up to a definite threshold, an explosive instability occurs near the zero line of the magnetic field. Part of the magnetic field energy is transformed into kinetic energy of the current sheet plasma, ejected with up to relativistic velocities. Meanwhile the dissipated magnetic field is regenerated by continued twisting, which ensures the recurrence of explosions. The origin of the explosions is connected with the interaction of the rotation and the magnetic field. That is why I call such explosions magnetorotational.

Now I would like to dwell on the most important features of the mechanism of magneto-rotational explosions (for more details, see Ozernoy and Somov, 1971).

The formation of the zero line of a magnetic field together with the corresponding current sheet is necessary for the appearance of such an instability. Let us consider the change of the geometry of the outer magnetic field in the course of contraction of the cloud with the initially homogeneous magnetic field perpendicular to the 
rotation axis. Let the rotation initially be small and insignificant. As shown in Figure 1, the first zero line of the X-type which has the form of a circle appears when contraction by a factor of $\sqrt{ } 3$ has already taken place. Figure 2 shows that the dissipation of the magnetic field takes place near the zero line and as the contraction continues this dissipation diminishes the number of force lines, going to infinity.

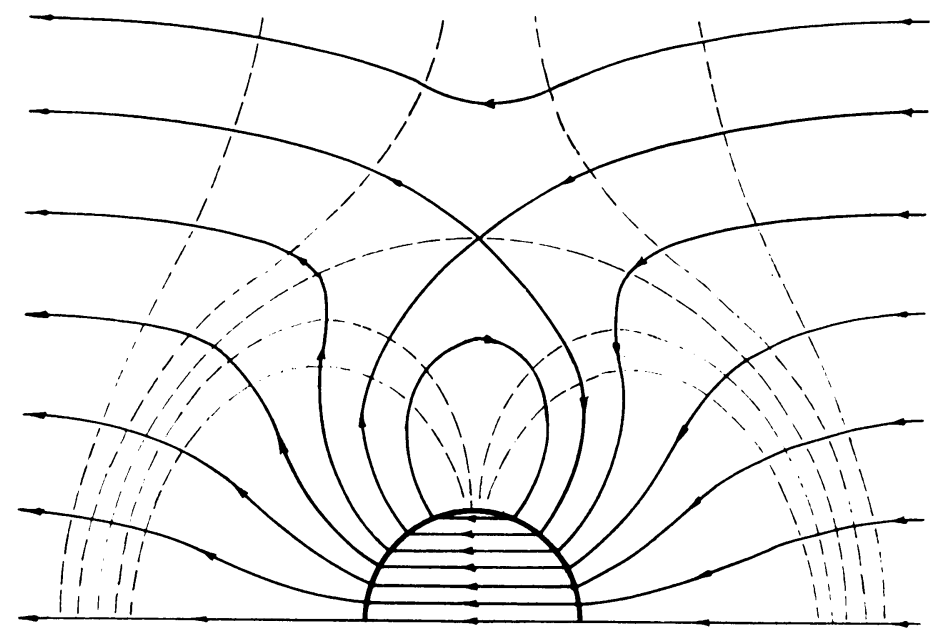

Fig. 1. The external magnetic field of the contracting cloud (solid lines) and the corresponding magnetic equipotentials (dashed lines).

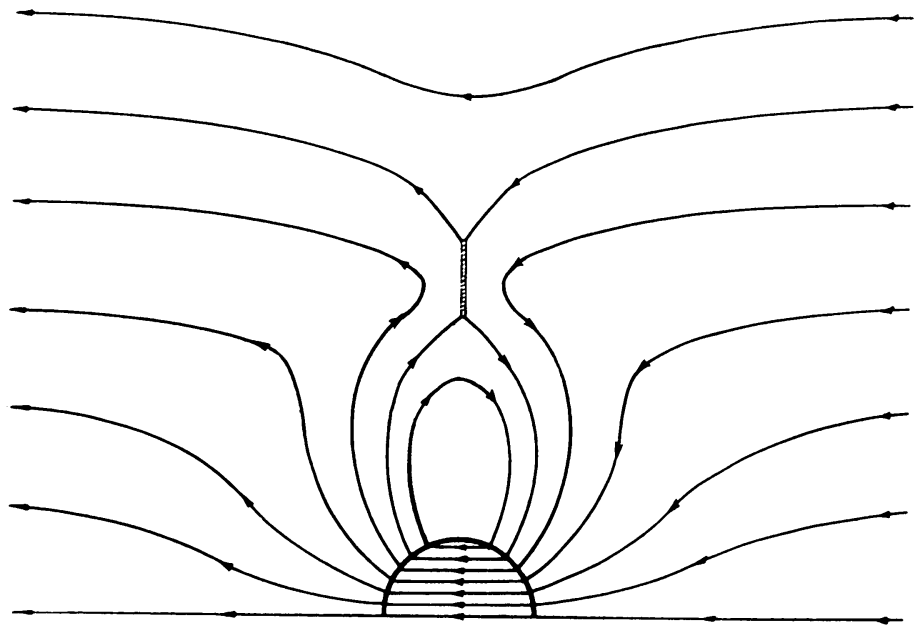

Fig. 2. The current sheet in the vicinity of the zero line of a magnetic field.

The process of cloud contraction is non-homologous, because the inner parts contract quicker than the outer ones. This leads to a more rapid increase of the radial component $H_{r}$ compared with the transverse one $H_{\theta}$. As a result, as shown in Figure 3, in the course of contraction the field acquires a predominantly radial character. 


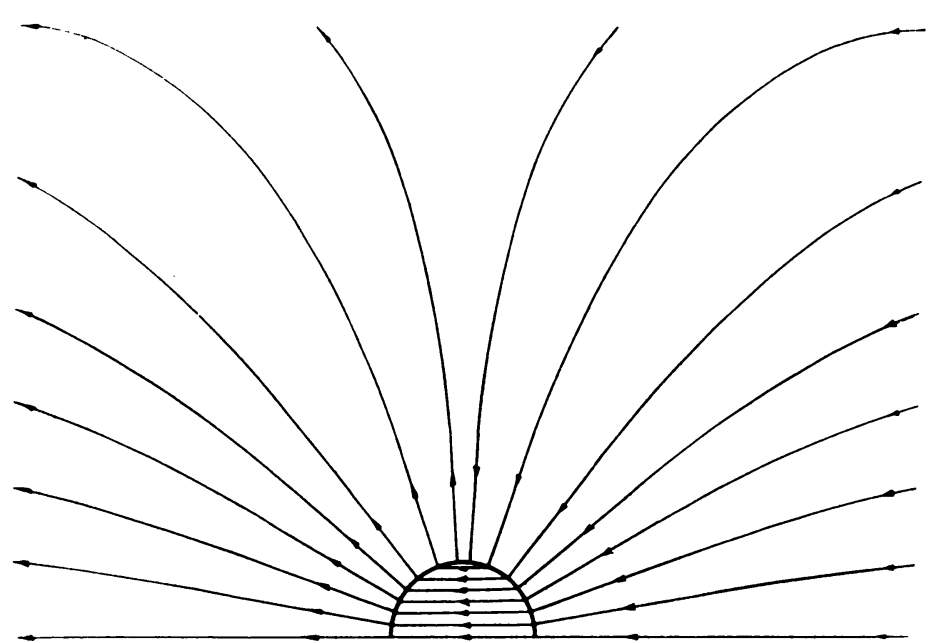

Fig. 3. The quasi-radial field forming due to the non-homologous character of a contraction.

The cloud contraction is brought to a stop by the growth of the pressure gradient and by the rotation, leading to the formation of a stationary magnetoid. In the intermediate stage of transition from contraction to equilibrium one or more bounces take place. As was suggested independently by Piddington (1966) and Ozernoy $(1966,1968)$ these bounces can lead to explosions producing clouds of relativistic electrons with a total energy characteristic of radio galaxies. The energy released is the magnetic energy which has been increased during the gravitational contraction. This is why I call such explosions magneto-gravitational as distinct from magneto-rotational which appear in the later stages of equilibrium.

When a cloud reaches its equilibrium state, a stationary magnetoid is formed. Subsequent contraction of the magnetoid is quasi-static with the velocity determined by the loss of angular momentum.

At this stage of quasi-stationary equilibrium, rotation becomes important for the amplification of the magnetic field. During contraction because of conservation of angular momentum, the cloud atmosphere is forced into differential rotation given by

$$
v_{\varphi}=\Omega R^{2} /(r) \sin \theta \quad(r>R),
$$

where $R$ is the radius of the cloud, $r$ is the distance from the center, $\Omega$ is the angular velocity and $\theta$ is a polar angle. Such differential rotation will lead to a twisting of the quasi-radial field, so that a toroidal component, $H_{\varphi}$, will appear:

$$
H_{\varphi}=-4 \pi N(R / r)^{4} H_{R} \sin \theta \quad(r>R),
$$

where $N$ is the number of revolutions. The topology of the twisted magnetic field near the rotational axis is shown in Figure 4.* The curvature of the force lines on one

* It is of interest that the resulting magnetic field (which is parallel to the equator near the surface and roughly perpendicular in outer layers) is very similar to that inferred by Kinman (1970) from his observations of some variable quasars. 


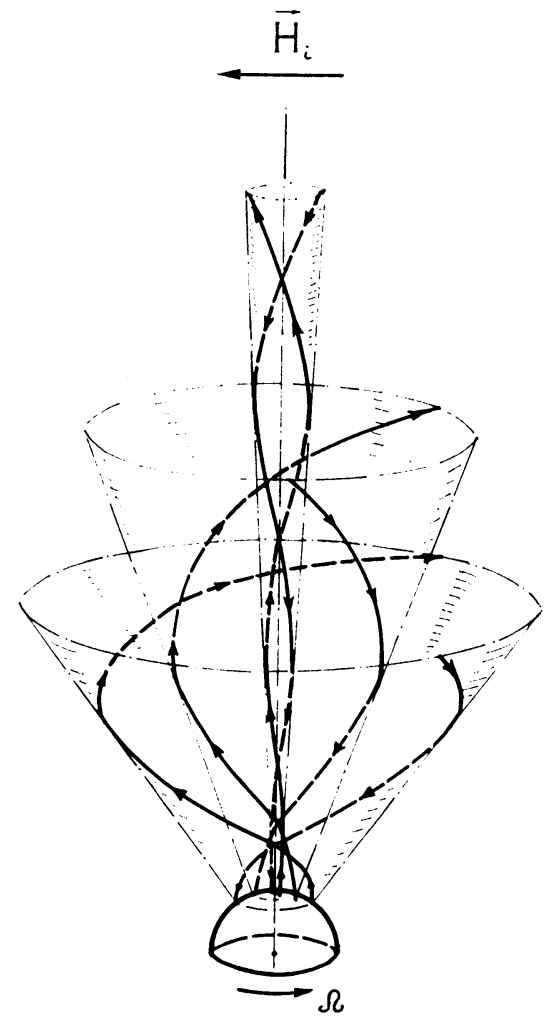

Fig. 4. The twisted magnetic field near the axis of rotation.

meridian decreases with approach to the rotational axis. Near the axis two force lines meet, oppositely directed and forming the zero line. Such a geometry offers the possibility of transforming the surplus magnetic energy, accumulated due to the rotation, into two symmetric ejections of accelerated particles, oppositely directed along the rotation axis. The acceleration may be conditioned by the mechanism of dynamic dissipation of a magnetic field (Syrovatsky, 1966). When the twisting of the magnetic field exceeds some threshold, the magnetic field near the zero line cannot be screened by quasi-stationary currents, but penetrates through the plasma into the zero line and prolongs the electric field $\mathbf{E} \sim \mathbf{H}$, which accelerates the current sheet plasma up to relativistic velocities.

The energy released by an explosion is of the order of the magnetic energy contained in the cylindrical canal surrounding the zero line. The radius of the canal

$$
r_{k} \approx v_{A} \tau
$$

Here $r_{k}$ is a cumulative radius, i.e. the radius within which the 'raking' of the magnetic field lines take place in the direction of the zero line as a result of the cumulative effect, and $\tau$ is the interval, during which the picture of the magnetic field is restored by the rotation after the previous explosion. 
For the energy of the explosion a simple formula is obtained

$$
\mathscr{E} \approx 10^{-2} H_{\varphi, e}^{2} R^{3}\left(r_{k} / R\right)^{4}
$$

where $H_{\varphi, e}$ is a toroidal magnetic field at the equator. The time interval between the successive explosions is determined by the expression

$$
\tau \approx 0.3\left(v / v_{A}\right)^{4 / 3} N^{-1 / 3} T
$$

where $v$ is the rotational velocity, $v_{A}$ is the Alfvén velocity in the magnetosphere, and $T=2 \pi\left(R^{3} / G M\right)^{1 / 2}$ is a rotation period in the regime of the plasma outflow from the equator.

Though the appearance of explosions is connected with the rotation, their periodicity does not coincide with the rotational period $T$, because the restoration of the dissipated magnetic energy by the rotation may be going on, generally speaking, both slower and quicker than for one revolution. When $N$ is small, $\tau \gg T$. With the growth of the number of revolutions, the value of $\tau / T$ decreases as $N^{-5 / 3}$ and becomes much less than unity, when the rotoidal field is large enough. The energy of explosions also decreases with time: $\mathscr{E} \sim N^{-2 / 3}$. Thus, magneto-rotational explosions become more 'feverish' and 'degenerate' with time.

As an example we shall estimate the parameters of explosions in the quasar nuclei. Let a gas conglomerate begin contraction with the following parameters:

$$
R_{i} \sim 10 \mathrm{pc} ; \quad \varrho_{i} \sim 10^{-16} \mathrm{~g} \mathrm{~cm}^{-3} ; \quad H_{i} \sim 3 \times 10^{-6} \mathrm{Oe} .
$$

As a result, a magnetoid is formed with mass $M \sim 3 \times 10^{9} M_{\odot}$ and with the following main parameters:

$$
L \sim 5 \times 10^{47} \mathrm{erg} \mathrm{s}^{-1} R \sim 10^{17} \mathrm{~cm}, T \sim 10 \mathrm{y} .
$$

These parameters are close to those for the nucleus of the quasar $3 C 273$.

In order to predict the parameters of the explosions, the theory uses two items of observational data: the age of the active phase, and the Alfvén velocity in the magnetosphere. These are derived from the movements in the emission line region, surrounding the nucleus (Greenstein and Schmidt, 1964) and from fluctuations of its optical variability (Ozernoy and Chertoprud, 1967). They are:

$$
t \sim 10^{4} \mathrm{y}, \quad v_{A} \sim 3 \times 10^{8} \mathrm{~cm} \mathrm{~s}^{-1} .
$$

Now we can find theoretically $N \sim 10^{3}, H_{R} \sim 3 \times 10^{-1} \mathrm{Oe}, H_{\varphi, e} \sim 3 \times 10^{3} \mathrm{Oe}, \tau \sim 3 \mathrm{yr}$, $r_{k} \sim 3 \times 10^{16} \mathrm{~cm}, \mathscr{E} \sim 10^{54} \mathrm{erg}$. These values prove to be in a rather good agreement with the observations available, including the time interval between successive radio bursts and the energy of a single burst calculated from the picture of radio variability.

The frequency spectrum, time variations and polarization of the flux of synchrotron radio emission from two clouds of relativistic electrons originating in a burst and flying apart in opposite directions with relativistic velocities, at the same time expanding, are calculated in the paper by Ozernoy and Sazonov (1969).

In conclusion I would note briefly some additional points.

(1) According to the dynamic dissipation theory, electrons of high energies, up to 
$E \sim 10^{22} \mathrm{eV}$, radiating in the $\mathrm{X}$-ray and $\gamma$-ray regions, should be generated during a burst. Estimates of $\gamma$-fluxes are given by Shklovsky (1970).

(2) The cumulative mechanism of explosions proposed follows a pulse regime. However, a magnetoid serves also as a source of continuous ejection of relativistic particles generated by the plasma turbulence.

(3) The dynamic dissipation leads to the acceleration of the current sheet plasma up to relativistic energies. The total mass of this relativistic plasma is, of course, too small. But there is no need to search for a new source of energy for the acceleration of thermal plasma clouds. The same relativistic particles may serve as such a source. By collisions with a magnetized thermal plasma cloud they can accelerate this cloud with their pressure up to high velocities.

(4) This last mechanism for acceleration of gas masses could, probably, explain the high velocities of the dwarf companions of some galaxies (for instance, in chains) as a result of their acceleration at the protogalaxy stage caused by the activity of a magnetoid in the parent galaxy.

\section{References}

Greenstein, J. L. and Schmidt, M.: 1964, Astrophys. J. 140, 1.

Kinman, T. D.: 1970, this volume, p. 164.

Ozernoy, L. M.: 1966, Astr. Zh. 43, 300.

Ozernoy, L. M.: 1968, in Problems of Stellar Evolution and Variable Stars, Symposium, Moscow, 24-27 Nov. 1964, (in Russian), "Nauka” Pub. House, p. 140.

Ozernoy, L. M.: 1971, Astr. Zh. 48, in press.

Ozernoy, L. M. and Chertoprud, V. E.: 1967, Astron. Zh. 44, 537.

Ozernoy, L. M. and Sazonov, V. N.: 1969, Astrophys. Space Sci. 3, 395.

Ozernoy, L. M. and Somov, B. V.: 1971, Astrophys. Space Sci. 11, 264.

Ozernoy, L. M. and Usov, V. V.: 1971, Astrophys. Space Sci., in press.

Piddington, J. H.: 1966, Monthly Notices Roy. Astron. Soc. 133, 163.

Shklovsky, I. S.: 1970, Astron. Zh. 47, 742.

Syrovatsky, S. I.: 1966, Astron. Zh. 43, 340; Zh. Eksperim. Teor. Fiz. 50, 1133.

\section{Discussion}

Wray: At Northwestern University a student of mine, Harry Heckathorn, has carried out a comprehensive study of the velocity field of the ionized gas in M82. Our results, obtained with the 40-in. telescope of the Lindheimer Astronomical Research Center, are consistent with earlier observations by E. M. Burbidge, and by Lynds and Sandage, at points observed in common. We find that, out to the limits of our observations, some $45 \mathrm{arc} \mathrm{sec}$ from the major axis, the ionized hydrogen is both expanding and rotating. Since it seems likely that the mechanical energy dominates the magnetic field in the regions we have observed, it would appear that we may have evidence for the twisting of any magnetic field which might be present, much in the way you require. It would be interesting to see if your theory may indeed be applicable to the problem of M82 and, if so, it would be interesting to see if there are any parameters common to both theory and our observations which could be investigated for agreement or disagreement, as the case may be. 